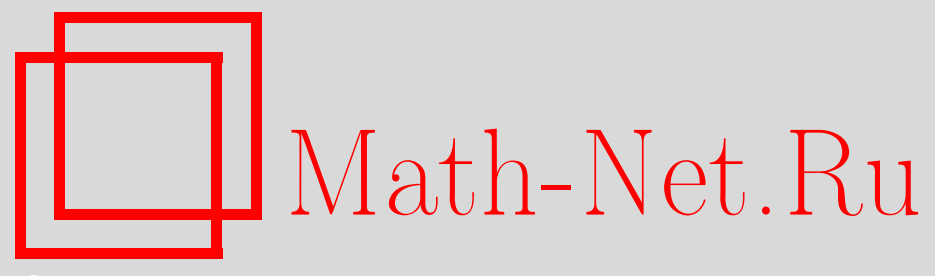

В. Г. Михайлов, Изучение предельного поведения числа решений систем уравнений со случайным вхождением неизвестных, Матем. вопр. криптогр., 2010, том 1, выпуск 3, 27-43

DOI: https://doi.org/10.4213/mvk14

Использование Общероссийского математического портала Math-Net.Ru подразумевает, что вы прочитали и согласны с пользовательским соглашением http://www.mathnet.ru/rus/agreement

Параметры загрузки:

IP : 18.209 .158 .208

26 апреля 2023 г., $18: 26: 19$ 


\author{
МАТЕМАТИЧЕСКИЕ ВОПРОСЫ КРИПТОГРАФИИ \\ 2010 T. 1 № 3 C. 27-43
}

УДК: 519.212 .2

\title{
Изучение предельного поведения числа решений систем уравнений со случайным вхождением неизвестных
}

\author{
В. Г. Михайлов \\ Математический институт им. В. А. Стеклова РАН, Москва
}

Получено 22.IV.2010

Рассматриваются заведомо совместные системы уравнений со случайным вхождением двоичных неизвестных по схеме неравновероятного выбора без возвращения. Указаны условия выполнения предельной теоремы Пуассона для двоичного логарифма числа решений системы при неограниченном увеличении числа неизвестных и числа уравнений.

Ключевые слова: случайные системы уравнений, число решений, предельная теорема Пуассона

On the limit behaviour of the number of solutions for compatible systems of equations with random selection of unknowns

\section{G. Mikhailov}

Steklov Mathematical Institute of RAS, Moscow

\begin{abstract}
Compatible systems of equations with random selection of binary unknowns are investigated. We consider the case of regular non-uniform polynomial selection of unknowns. The sufficient conditions for the weak convergence of the logarithm of the number of solutions to the Poisson law are found.
\end{abstract}

Key words: random systems of equations, number of solutions, Poisson limit theorem

Citation: Mathematical Aspects of Cryptography, 2010, vol. 1, no. 3, pp. 27-43 (Russian). 


\section{1. Введение}

Пусть $\varphi_{t}\left(u_{1}, \ldots, u_{d(t)}\right), t=1,2, \ldots,-$ некоторые функции от двоичных переменных со значениями в некотором множестве $A$. Будем считать, что они существенно зависят от всех своих аргументов. Рассмотрим систему случайных уравнений

$$
\varphi_{t}\left(x_{j_{1}(t)}, \ldots, x_{j_{d(t)}(t)}\right)=\varphi_{t}\left(x_{j_{1}(t)}^{0}, \ldots, x_{j_{d(t)}(t)}^{0}\right), \quad t=1, \ldots, T,
$$

относительно неизвестных $x_{1}, \ldots, x_{N} \in\{0,1\}$, где индексы $j_{i}(t)$ выбраны с помощью случайного выбора без возвращения из множества $\{1, \ldots, N\}$ (проводимого независимо и, возможно, по своему правилу для каждого уравнения; точные определения даны ниже), а $x^{0}=\left(x_{1}^{0}, \ldots, x_{N}^{0}\right)-$ некоторый набор чисел из $\{0,1\}$, представляющий собой заданное заранее решение системы.

Нас интересует асимптотическое поведение распределения числа решений системы (1) при согласованном стремлении параметров $T$ и $N$ к бесконечности и не зависящих от $T$ функциях $\varphi_{t}\left(u_{1}, \ldots, u_{d(t)}\right)$. Метод изучения асимптотических свойств этого распределения был предложен Г. В.Балакиным [1] и разработан В.А. Копытцевым в [2]. В работе [2] рассматривался случай, когда все функции $\varphi_{t}\left(u_{1}, \ldots, u_{d(t)}\right)$ одинаковы, а наборы индексов $j_{i}(t)$ выбираются равновероятно. Были указаны условия, при которых распределение двоичного логарифма числа решений системы (1) сходится к пуассоновскому распределению. Случай, когда функции $\varphi_{t}\left(u_{1}, \ldots, u_{d(t)}\right)$ различны, но числа $d(t)$ ограничены при переходе к пределу, рассматривался в работах [3] и [4].

Настоящая работа является второй частью исследования, цель которого - обобщение упомянутых результатов на случай неравновероятного выбора неизвестных в уравнениях. Для достижения этой цели в работе [5] был разработан специальный метод. Суть его состоит в сведении решаемой задачи к задаче об асимптотических свойствах распределения числа пустых ячеек в схеме размещения частиц комплектами.

Опишем способ формирования системы (1), рассмотренный в работе [5]. Пусть подмножества $\mathcal{B}_{1}, \ldots, \mathcal{B}_{T} \subseteq\{1, \ldots, N\}$ выбраны случайно и независимо в соответствии с некоторыми распределениями $P_{1}, \ldots, P_{T}$ на множестве всех $d$-подмножеств множества $\{1, \ldots, N\}$. Множество $\mathcal{B}_{t}$ представляет собой набор номеров неизвестных, которые входят в $t$-е уравнение системы. В постановке работы [5] порядок их использования в качестве аргументов функции может быть произвольным. Единственное условие - он не зависит от выбора и расстановки неизвестных в других уравнениях. Такая 
схема выбора и расстановки неизвестных в [5] была названа общей схемой (или общей постановкой задачи).

В частном случае, когда подмножества $\mathcal{B}_{1}, \ldots, \mathcal{B}_{T}$ имеют равномерное распределение на множестве всех $d$-подмножеств $\{1, \ldots, N\}$, порядок использования их элементов в качестве индексов у аргументов функции случаен, распределен равновероятно и независимо для каждого уравнения, а функции $\varphi_{t}\left(u_{1}, \ldots, u_{d(t)}\right)$ одинаковы, получаем постановку работы [2]. Если же эти функции различны, получаем постановку работ [3] и [4].

В данной работе рассматривается случай, когда $d(1)=d(2)=\ldots=d$, а набор индексов $j_{1}(t), \ldots, j_{d}(t)$ выбирается из множества

$$
K_{d}=\left\{\left(k_{1}, \ldots, k_{d}\right) \in\{1, \ldots, N\}^{d}: k_{i} \neq k_{j}(i \neq j)\right\}
$$

случайно с вероятностями (они, вообще говоря, зависят от $t$ )

$$
P_{t}\left(k_{1}, \ldots, k_{d}\right)=\mathbf{P}\left\{\left(j_{1}(t), \ldots, j_{d}(t)\right)=\left(k_{1}, \ldots, k_{d}\right)\right\}, \quad t=1, \ldots, T
$$

При этом $P_{t}\left(k_{1}, \ldots, k_{d}\right)=0,\left(k_{1}, \ldots, k_{d}\right) \notin K_{d}$.

Пусть для этих вероятностей выполнены условия

$$
P_{t}\left(k_{1}, \ldots, k_{d}\right)=p_{t, k_{1}} p_{t, k_{2}} \ldots p_{t, k_{d}}\left(1+\beta_{t}\left(k_{1}, \ldots, k_{d}\right)\right)
$$

где $\sum_{k=1}^{N} p_{t, k}=1$,

$$
\begin{gathered}
\left|\beta_{t}\left(k_{1}, \ldots, k_{d}\right)\right| \leq \frac{C}{N}, \quad\left(k_{1}, \ldots, k_{d}\right) \in K_{d}, \quad t=1, \ldots, T, \\
\frac{c}{N} \leq p_{t, k} \leq \frac{C}{N}, \quad k=1,2, \ldots, N, \quad t=1,2, \ldots, T,
\end{gathered}
$$

при некоторых $0<c<C<\infty$. Такой способ выбора и расстановки неизвестных в уравнениях системы (1) назовем регулярным неоднородным полиномиальным выбором без возвращения.

Частным случаем является обычный равновероятный выбор без возвращения. Для него

$$
p_{t, k_{1}}=p_{t, k_{2}}=\ldots=p_{t, k_{d}}=\frac{1}{N}
$$

Действительно, в этом случае

$$
P_{t}\left(k_{1}, \ldots, k_{d}\right)=\frac{1}{(N)_{d}}=\frac{1}{N^{d}}\left(1+\beta_{t}\left(k_{1}, \ldots, k_{d}\right)\right),
$$


где при $N \geq(d+1) d$

$$
0<\beta_{t}\left(k_{1}, \ldots, k_{d}\right)=\prod_{s=0}^{d-1}\left(1-\frac{s}{N}\right)^{-1}-1 \leq \frac{d^{2}}{N} .
$$

При изучении системы с регулярным неоднородным полиномиальным выбором неизвестных наложим на решение $x^{0}=\left(x_{1}^{0}, \ldots, x_{N}^{0}\right)$ системы (1) условия, аналогичные использованным В. А. Копытцевым в работе [2]. Пусть $J_{0}-$ множество нулевых координат, $n_{0}=\left|J_{0}\right|$ - число нулей, $J_{1}-$ множество единичных координат, а $n_{1}=\left|J_{1}\right|$ - число единиц в записи решения $x^{0}=\left(x_{1}^{0}, \ldots, x_{N}^{0}\right)$. (Здесь и далее число элементов конечного множества $F$ будем обозначать $|F|$.) Считаем, что существуют константы $0<c_{0}<c_{1}<1$, при которых для всех достаточно больших $N$

$$
c_{0} \leq \frac{n_{1}}{N}=1-\frac{n_{0}}{N} \leq c_{1} .
$$

Итак, мы исследуем асимптотическое поведение при $N, T \rightarrow \infty$ распределения числа решений заведомо совместной системы уравнений

$$
\varphi_{t}\left(x_{j_{1}(t)}, \ldots, x_{j_{d}(t)}\right)=\varphi_{t}\left(x_{j_{1}(t)}^{0}, \ldots, x_{j_{d}(t)}^{0}\right), \quad t=1, \ldots, T,
$$

относительно неизвестных $x_{1}, \ldots, x_{N} \in\{0,1\}$, где индексы $j_{i}(t)$ выбраны с помощью случайного регулярного неоднородного полиномиального выбора без возвращения из множества $\{1, \ldots, N\}$, удовлетворяющего условиям (2)-(4), а $x^{0}=\left(x_{1}^{0}, \ldots, x_{N}^{0}\right)$ - заданное заранее решение системы, удовлетворяющее условию (5). Здесь $\varphi_{t}\left(u_{1}, \ldots, u_{d}\right), t=1,2, \ldots,-$ фиксированная последовательность функций от двоичных векторов со значениями в некотором множестве $A$. Они существенно зависят от всех своих аргументов и не меняются при переходе к пределу по $T$.

Мы рассмотрим два исключающих друг друга случая, описываемых следующими условиями.

Условие $(A)$. При всех $T$ среди функций $\varphi_{t}, t=1,2, \ldots, T$, найдется не менее $M(T)$ функций, обладающих следующим свойством:

$$
\varphi_{t}\left(y_{1}, \ldots, y_{d}\right) \neq \varphi_{t}\left(1-y_{1}, \ldots, 1-y_{d}\right)
$$

для некоторого вектора $y=\left(y_{1}, \ldots, y_{d}\right) \in V^{d}$ (вектор $y$ при каждом $t$ может быть свой). Функция $M(T)$ обладает свойством $M(T) \ln T / T \rightarrow \infty$ при $T \rightarrow \infty$. 
Условие $(B)$. При всех $t$ и всех $y=\left(y_{1}, \ldots, y_{d}\right) \in V^{d}$

$$
\varphi_{t}\left(y_{1}, \ldots, y_{d}\right)=\varphi_{t}\left(1-y_{1}, \ldots, 1-y_{d}\right) .
$$

Случайное множество индексов неизвестных, не входящих в левые части уравнений системы (6), обозначим через $\mathcal{M}_{0}$. Будем говорить, что переменная $x_{i}$ допускает варьирование в $t$-м уравнении, если она входит в него, и при изменении ее значения в векторе $\left(x_{1}^{0}, \ldots, x_{N}^{0}\right)$ на противоположное мы снова получим решение $t$-го уравнения. Множество индексов неизвестных, которые присутствуют в системе и допускают варьирование во всех уравнениях, в которые входят, обозначим через $\mathcal{N}$.

Пусть $S$ - множество решений системы (6), $\xi=|S|$,

$$
D=\left\{\left(x_{1}, \ldots, x_{N}\right) \in\{0,1\}^{N}: x_{i}=x_{i}^{0} \quad \forall i \notin \mathcal{M}_{0} \cup \mathcal{N}\right\} .
$$

Решения системы, принадлежащие множеству $D$, будем называть близкими к решению $x^{0}$. Обозначим их число $\xi^{\prime}=|S \cap D|$. Остальные решения назовем посторонними решениями системы (6).

В работе [5] было доказано, что для системы (6) с регулярным неоднородным полиномиальным выбором неизвестных без возвращения при естественных условиях (все они учтены в формулировках теорем 1 и 2 ниже) выполнено соотношение

$$
\mathbf{P}\left\{\xi^{\prime}=2^{k}\right\}-\frac{\lambda_{T, N}^{k}}{k !} e^{-\lambda_{T, N}} \rightarrow 0, \quad k=0,1, \ldots,
$$

где

$$
\lambda_{T, N}=\sum_{k=1}^{N} \exp \left\{-\sum_{t=1}^{T} a_{t, k}\right\},
$$

a $a_{t, k}$ - вероятность того, что переменная $x_{k}$ присутствует и не допускает варьирования в $t$-м уравнении (формулы для этих величин приведены ниже в $§ 2)$.

В настоящей работе доказываются следующие утверждения.

Теорема 1. Пусть $d(1)=d(2)=\ldots=d$, параметры $T, N \rightarrow \infty$, причем $T=O(N \ln N)$, выполнены условия (2)-(5), $\mathbf{E} \xi=O(1) u$

$$
\mathbf{E}\left(\left|\mathcal{M}_{0}\right|+|\mathcal{N}|\right)=O(1) .
$$

Тогда:

если выполнено условие $(A)$, то $\mathbf{E} \xi-\mathbf{E} \xi^{\prime} \rightarrow 0$ и $\mathbf{P}\left\{\xi=\xi^{\prime}\right\} \rightarrow 1$;

если выполнено условие $(B)$, то $\mathbf{E} \xi-2 \mathbf{E} \xi^{\prime} \rightarrow 0 u \mathbf{P}\left\{\xi=2 \xi^{\prime}\right\} \rightarrow 1$. 
Теорема 2. Пусть выполнены условия теоремы 1 и существует предел $\mu=\lim \lambda_{T, N}, 0<\mu<\infty$. Тогда:

если выполнено условие $(A)$, то

$$
\mathbf{P}\left\{\xi=2^{k}\right\} \rightarrow \frac{\mu^{k}}{k !} e^{-\mu}, \quad k=0,1, \ldots,
$$

если выполнено условие (B), то

$$
\mathbf{P}\left\{\xi=2^{k+1}\right\} \rightarrow \frac{\mu^{k}}{k !} e^{-\mu}, \quad k=0,1, \ldots
$$

ЗАМЕчАНИЕ 1. Из условий теоремы следует, что $N \ln N=O(T)$ (см. замечание 5 ниже).

Теорема 2 распространяет предельную теорему работы [2] для числа решений систем уравнений со случайным вхождением двоичных неизвестных на случай регулярного неоднородного полиномиального выбора неизвестных в уравнениях.

\section{2. Некоторые определения и обозначения}

Введем обозначение $V^{d}$ для множества всех векторов $d$-мерного линейного пространства над полем $\mathrm{GF}(2)$, через $\|y\|$ обозначим число ненулевых координат вектора $y$, а через $\delta_{s}$ обозначим вектор из $V^{d}$, имеющий единицу в качестве $s$-й координаты и нули в качестве остальных координат. Рассмотрим индикаторную функцию вектора $y \in V^{d}$

$$
I_{t, s}(y)=I\left\{\varphi_{t}(y) \neq \varphi_{t}\left(y+\delta_{s}\right)\right\} .
$$

Обозначим через $|y|_{s, 0}$ и $|y|_{s, 1}$ число нулей и число единиц в записи вектора $y=\left(y_{1}, \ldots, y_{d}\right)$ без учета компоненты $y_{s}$. Положим

$$
L_{t, s}(y)=\left(\sum_{k \in J_{0}} p_{t, k}\right)^{|y|_{s, 0}}\left(\sum_{k \in J_{1}} p_{t, k}\right)^{|y|_{s, 1}} .
$$

Теперь мы можем выписать формулу для вероятностей $a_{t, k}$, входящих в формулу (8). Согласно нашим определениям

$$
a_{t, k}=\sum_{s=1}^{d} \sum_{y \in V^{d}: y_{s}=x_{k}^{0}} \sum_{\substack{k_{l} \in J_{y_{l}}, l=1, \ldots, s-1, s+1, \ldots, d}} I_{t, s}(y) P_{t}\left(k_{1}, \ldots, k_{s-1}, k, k_{s+1}, \ldots, k_{d}\right) .
$$


Используя (2)-(4) и (13), получаем, что при $N \rightarrow \infty$

$$
\begin{gathered}
a_{t, k}=p_{t, k} \sum_{s=1}^{d} \sum_{y \in V^{d}: y_{s}=x_{k}^{0} k_{l} \in J_{y_{l}, l=1, \ldots, s-1, s+1, \ldots, d}} I_{t, s}(y) L_{t, s}(y)\left(1+O\left(\frac{1}{N}\right)\right)= \\
=\frac{p_{t, k}}{2} \sum_{s=1}^{d} \sum_{y \in V^{d}} \sum_{k_{l} \in J_{y_{l}}, l=1, \ldots, s-1, s+1, \ldots, d} I_{t, s}(y) L_{t, s}(y)\left(1+O\left(\frac{1}{N}\right)\right)= \\
=p_{t, k} L_{t}\left(1+O\left(\frac{1}{N}\right)\right),
\end{gathered}
$$

где

$$
L_{t}=\sum_{s=1}^{d} L_{t, s}, \quad L_{t, s}=\frac{1}{2} \sum_{y \in V^{d}} I_{t, s}(y) L_{t, s}(y) .
$$

Введем обозначения, которые понадобятся нам в следующем разделе. Пусть

$$
\begin{aligned}
J_{\delta}\left(x^{1}\right) & =J_{\delta} \cap\left\{k: x_{k}^{1}=x_{k}^{0}\right\}, \quad \delta \in\{0,1\}, \\
L_{t, s}\left(y, x^{1}\right) & =\left(\sum_{k \in J_{0}\left(x^{1}\right)} p_{t, k}\right)^{|y|_{s, 0}}\left(\sum_{k \in J_{1}\left(x^{1}\right)} p_{t, k}\right)^{|y|_{s, 1}} .
\end{aligned}
$$

Положим

$$
w=w\left(x^{1}\right)=\left|\left\{k: x_{k}^{1} \neq x_{k}^{0}\right\}\right| .
$$

Из условий (4) и (5) следует, что

$$
\sum_{k \in J_{0}} p_{t, k} \geq c\left(1-c_{1}\right), \quad \sum_{k \in J_{1}} p_{t, k} \geq c c_{0}
$$

Поэтому при $N \rightarrow \infty$

$$
L_{t, s}\left(y, x^{1}\right)=L_{t, s}(y)\left(1+O\left(\frac{w}{N}\right)\right)
$$

равномерно по $t, s$ и $x^{1}$.

ЗАмечАниЕ 2. В силу (2)-(5) сумма $\sum_{k: x_{k}^{0} \neq x_{k}^{1}} p_{t, k}=O\left(\frac{w}{N}\right)$ равномерно по $t$ при $N \rightarrow \infty$ (величина

ЗАМЕЧАНИЕ 3 . Для любой функции на $d$-мерном двоичном кубе, отличной от константы, найдутся две смежные вершины, на которых функция принимает разные значения. Поэтому $I_{t, s}(y)=1$ при некоторых $y$ и $s$. 
Согласно условиям (2)-(5) распределение $d$-мерного случайного вектора $\eta_{t}^{0}$ при достаточно больших $N$ положительно во всех точках такого куба. Поэтому из (16), (18), (19) следует, что $c^{\prime} \leq L_{t} \leq c^{\prime \prime}, \quad t=1, \ldots, T$, где $0<c^{\prime}<c^{\prime \prime}<\infty$.

\section{3. Вспомогательные оценки}

Пусть $P_{t}\left(x^{1}\right)$ - вероятность того, что вектор $x^{1}=\left(x_{1}^{1}, \ldots, x_{N}^{1}\right)$ является решением $t$-го уравнения. Настоящий раздел посвящен выводу оценок для величин $P_{t}\left(x^{1}\right)$.

Лемма 1. Пусть выполнены условия (2)-(5). Тогда

$$
\left|P_{t}\left(x^{1}\right)-1+L_{t} \sum_{k: x_{k}^{0} \neq x_{k}^{1}} p_{t, k}\right| \leq C^{\prime} \frac{w}{N} \sum_{k: x_{k}^{1} \neq x_{k}^{0}} p_{t, k} \leq C^{\prime \prime}\left(\frac{w}{N}\right)^{2}
$$

с константами $C^{\prime}, C^{\prime \prime}<\infty$. Величины $L_{t}$ и $=w\left(x^{1}\right)$ определены формулами (15) и (17) соответственно.

Из (20) следует, что при $N \rightarrow \infty$ равномерно по $t$ и $x^{1}$

$$
\begin{aligned}
& P_{t}\left(x^{1}\right)=1-L_{t} \sum_{k: x_{k}^{0} \neq x_{k}^{1}} p_{t, k}+O\left(\frac{w}{N}\right)^{2}= \\
& =\left(1-L_{t} \sum_{k: x_{k}^{0} \neq x_{k}^{1}} p_{t, k}\right)\left(1+O\left(\frac{w}{N}\right)^{2}\right) .
\end{aligned}
$$

Так как при переходе к пределу величины $L_{1}, \ldots, L_{T}$ отделены от нуля (см. замечание 3 ), то из (20) следует, что при некоторой константе $\tilde{C}<\infty$

$$
P_{t}\left(x^{1}\right) \leq 1-L_{t}\left(1-\tilde{C} \frac{w}{N}\right) \sum_{k: x_{k}^{0} \neq x_{k}^{1}} p_{t, k} .
$$

Доказательство леммы 1. Заметим, что

$$
1-P_{t}\left(x^{1}\right)=\mathbf{P}\left\{\varphi_{t}\left(\eta_{t}^{1}\right) \neq \varphi_{t}\left(\eta_{t}^{0}\right)\right\}=P_{t}^{\prime}\left(x^{1}\right)+P_{t}^{\prime \prime}\left(x^{1}\right),
$$

где

$$
\begin{gathered}
\eta_{t}^{0}=\left(x_{j_{1}(t)}^{0}, \ldots, x_{j_{d}(t)}^{0}\right), \quad \eta_{t}^{1}=\left(x_{j_{1}(t)}^{1}, \ldots, x_{j_{d}(t)}^{1}\right), \\
P_{t}^{\prime}\left(x^{1}\right)=\mathbf{P}\left\{\varphi_{t}\left(\eta_{t}^{1}\right) \neq \varphi_{t}\left(\eta_{t}^{0}\right),\left\|\eta_{t}^{1}-\eta_{t}^{0}\right\|=1\right\}, \\
P_{t}^{\prime \prime}\left(x^{1}\right)=\mathbf{P}\left\{\varphi_{t}\left(\eta_{t}^{1}\right) \neq \varphi_{t}\left(\eta_{t}^{0}\right),\left\|\eta_{t}^{1}-\eta_{t}^{0}\right\| \geq 2\right\} .
\end{gathered}
$$


Тогда

$$
1-P_{t}\left(x^{1}\right)=P_{t}^{\prime}\left(x^{1}\right)+P_{t}^{\prime \prime}\left(x^{1}\right) .
$$

Согласно определению

$$
P_{1}=\sum_{y \in V^{d}} \sum_{s=1}^{d} I_{t, s}(y) \mathbf{P}\left\{\eta_{t}^{0}=y, \eta_{t}^{1}=y+\delta_{s}\right\} .
$$

Используя (24), (19), (2)-(5), получаем

$$
\begin{gathered}
\mathbf{P}\left\{\eta_{t}^{0}=y, \eta_{t}^{1}=y+\delta_{s}\right\}= \\
\sum_{k_{1} \in J_{y_{1}}\left(x^{1}\right), \ldots, k_{s-1} \in J_{y_{s-1}}\left(x^{1}\right), k_{s} \notin J_{y_{s}}\left(x^{1}\right), k_{s+1} \in J_{y_{s+1}}\left(x^{1}\right), \ldots, k_{d} \in J_{y_{d}}\left(x^{1}\right)} P_{t}\left(k_{1}, \ldots, k_{d}\right)= \\
=L_{t, s}\left(y, x^{1}\right) \sum_{k: x_{k}^{0}=y_{s}, x_{k}^{1}=1-y_{s}} p_{t, k}\left(1+O\left(\frac{1}{N}\right)\right)= \\
=L_{t, s}(y) \sum_{k: x_{k}^{0}=y_{s}, x_{k}^{1}=1-y_{s}} p_{t, k}\left(1+O\left(\frac{w}{N}\right)\right), \quad N \rightarrow \infty
\end{gathered}
$$

равномерно по $t, s$ и $x^{1}$.

Так как $I_{t, s}(y)=I_{t, s}\left(y+\delta_{s}\right)$ и $L_{t, s}(y)=L_{t, s}\left(y+\delta_{s}\right)$ (сложение векторов производится над полем $\mathrm{GF}(2))$, то, используя (24), получаем равенства

$$
\begin{gathered}
I_{t, s}(y) \mathbf{P}\left\{\eta_{t}^{0}=y, \eta_{t}^{1}=y+\delta_{s}\right\}+ \\
+I_{t, s}\left(y+\delta_{s}\right) \mathbf{P}\left\{\eta_{t}^{0}=y+\delta_{s}, \eta_{t}^{1}=y\right\}= \\
=I_{t, s}(y) L_{t, s}(y) \sum_{k: x_{k}^{0} \neq x_{k}^{1}} p_{t, k}\left(1+O\left(\frac{w}{N}\right)\right)
\end{gathered}
$$

и

$$
P_{1}=L_{t} \sum_{k: x_{k}^{0} \neq x_{k}^{1}} p_{t, k}\left(1+O\left(\frac{w}{N}\right)\right),
$$

где $L_{t}$ определены формулами (15).

Оценим теперь величину $P_{t}^{\prime \prime}\left(x^{1}\right)$. Из определений и свойств (2)-(4) следует, что

$$
P_{t}^{\prime \prime}\left(x^{1}\right) \leq \mathbf{P}\left\{\left\|\eta_{t}^{1}-\eta_{t}^{0}\right\| \geq 2\right\} \leq \sum_{1 \leq u<v \leq d} \mathbf{P}\left\{x_{j_{u}}^{1} \neq x_{j_{u}}^{0}, x_{j_{v}}^{1} \neq x_{j_{v}}^{0}\right\},
$$


a

$$
\begin{gathered}
\quad \mathbf{P}\left\{x_{j_{u}}^{1} \neq x_{j_{u}}^{0}, x_{j_{v}}^{1} \neq x_{j_{v}}^{0}\right\}=\sum_{k_{u}: x_{k_{u}}^{1} \neq x_{k_{u}}^{0}, k_{v}: x_{k_{v}}^{1} \neq x_{k_{v}}^{0}} P_{t}\left(k_{1}, \ldots, k_{d}\right) \leq \\
\leq C_{1} \sum_{k_{u}: x_{k_{u}}^{1} \neq x_{k_{u}}^{0}, k_{v}: x_{k_{v}}^{1} \neq x_{k_{v}}^{0}} p_{t, k_{u}} p_{t, k_{v}} \leq C_{2} \frac{w}{N} \sum_{k: x_{k}^{1} \neq x_{k}^{0}} p_{t, k} \leq C_{3}\left(\frac{w}{N}\right)^{2},
\end{gathered}
$$

где $C_{1}, C_{2}, C_{3}<\infty$. Поэтому

$$
P_{t}^{\prime \prime}\left(x^{1}\right) \leq C_{4} \frac{w}{N} \sum_{k: x_{k}^{1} \neq x_{k}^{0}} p_{t, k} \leq C_{5}\left(\frac{w}{N}\right)^{2}
$$

при некоторых константах $C_{4}, C_{5}<\infty$.

Используя (23), (26), (27), получаем неравенства (20). Лемма 1 доказана.

Лемма 2. Пусть выполнены условия (2)-(5) и задано некоторое $\varepsilon \in$ $\in(0,1)$. Тогда найдутся такие числа $N(\varepsilon)<\infty$ и $c(\varepsilon)>0$, что при всех $t=1,2, \ldots$ всех $x^{1}$, удовлетворяющих неравенствам

$$
\varepsilon N \leq w\left(x^{1}\right) \leq(1-\varepsilon) N,
$$

при $N>N(\varepsilon)$ выполнено неравенство $P_{t}\left(x^{1}\right)<1-c(\varepsilon)$.

Доказательство леммы 2. При условии (28) выполняется хотя бы одна из четырех пар неравенств (при $\alpha, \beta \in\{0,1\})$ :

$$
\left|\left\{k: x^{0}=\alpha, x^{1}=1-\alpha\right\}\right|>\frac{\varepsilon N}{2}, \quad\left|\left\{k: x^{0}=\beta, x^{1}=\beta\right\}\right|>\frac{\varepsilon N}{2} .
$$

Пусть в (29) $\alpha=\beta=0$ (остальные случаи рассматриваются аналогично). Тогда воспользуемся тем, что функция $\varphi_{t}$ отлична от константы, а значит, найдется такой вектор $y=\left(y_{1}, \ldots, y_{d}\right) \in V^{d}$, что $\varphi_{t}\left(y_{1}, \ldots, y_{d}\right) \neq \varphi_{t}(0, \ldots, 0)$. Поэтому

$$
\begin{gathered}
1-P_{t}\left(x^{1}\right)=\mathbf{P}\left\{\varphi_{t}\left(\eta_{t}^{1}\right) \neq \varphi_{t}\left(\eta_{t}^{0}\right)\right\} \geq \\
\geq \mathbf{P}\left\{\eta_{t}^{1}=\left(y_{1}, \ldots, y_{d}\right), \eta_{t}^{0}=(0, \ldots, 0)\right\} .
\end{gathered}
$$

В свою очередь, из (29) при $\alpha=\beta=0$ и условий (2)-(5) следует, что вероятность события в правой части (30) при больших $N$ положительна и отделена от нуля. Лемма 2 доказана. 
Лемма 3. Пусть функичия $\varphi_{t}$ такова, что

$$
\varphi_{t}\left(y_{1}, \ldots, y_{d}\right) \neq \varphi_{t}\left(1-y_{1}, \ldots, 1-y_{d}\right)
$$

для некоторого вектора $\left(y_{1}, \ldots, y_{d}\right) \in V^{d}$. Пусть также выполнены условия (2)-(5) и задано некоторое $\varepsilon \in(0,1)$. Тогда найдутся такие числа $N^{\prime}(\varepsilon)<\infty$ u $c^{\prime}(\varepsilon)>0$, что $P_{t}\left(x^{1}\right)<1-c^{\prime}(\varepsilon)$ при $N>N^{\prime}(\varepsilon)$, всех $t=1,2, \ldots$ и всех $x^{1}$, для которых

$$
(1-\varepsilon) N<w\left(x^{1}\right) .
$$

Доказательство леммы 3 аналогично предыдущему. Согласно условиям леммы

$$
1-P_{t}\left(x^{1}\right) \geq \mathbf{P}\left\{\eta_{t}^{1}=\left(y_{1}, \ldots, y_{d}\right), \eta_{t}^{0}=\left(1-y_{1}, \ldots, 1-y_{d}\right)\right\} .
$$

Из (31) и условий (2)-(5) следует, что вероятность события в правой части (32) при больших $N$ положительна и отделена от нуля. Лемма 3 доказана.

\section{4. Основная лемма}

В основе доказательств теорем лежит следующее утверждение.

Лемма 4. Пусть выполнены условия теоремы 1. Если выполнено условие $(A)$, то при $N \rightarrow \infty$

$$
\mathbf{E} \xi=e^{\lambda_{T, N}\left(1+O\left(N^{-1}\right)\right)}+o(1) .
$$

Если же выполнено условие (В), то при $N \rightarrow \infty$

$$
\mathbf{E} \xi=2 e^{\lambda_{T, N}\left(1+O\left(N^{-1}\right)\right)}+o(1) .
$$

Здесь величины $\lambda_{T, N}$ выражаются формулой (8).

Доказательство леммы 4. Мы будем следовать схеме рассуждений, во многом аналогичной схеме, предложенной в работе [2]. Воспользуемся формулой

$$
\mathbf{E} \xi=\sum_{x^{1}} \prod_{t=1}^{T} P_{t}\left(x^{1}\right)=E_{1}+E_{2}+E_{3}+E_{4},
$$


где $P_{t}\left(x^{1}\right)$ - вероятность того, что вектор $x^{1}=\left(x_{1}^{1}, \ldots, x_{N}^{1}\right)$ является решением $t$-го уравнения, а величины $E_{1}, E_{2}, E_{3}, E_{4}$ представляют собой суммы слагаемых $\prod_{t=1}^{T} P_{t}\left(x^{1}\right)$ по множествам

$$
\begin{aligned}
& \Sigma_{1}=\left\{x^{1}: w\left(x^{1}\right) \leq N^{1 / 3}\right\}, \\
& \Sigma_{2}=\left\{x^{1}: N^{1 / 3}<w\left(x^{1}\right) \leq \varepsilon N\right\}, \\
& \Sigma_{3}=\left\{x^{1}: \varepsilon N \leq w\left(x^{1}\right) \leq(1-\varepsilon) N\right\}, \\
& \Sigma_{4}=\left\{x^{1}: w\left(x^{1}\right)>(1-\varepsilon) N\right\}
\end{aligned}
$$

соответственно.

Число $\varepsilon \in(0,1)$ будет выбрано позже.

Оценим сумму $E_{1}$. Используя (21), равенство $P_{t}\left(x^{0}\right)=1$ и условия $T=O(N \ln N), w \leq N^{1 / 3}$, получаем

$$
\begin{aligned}
E_{1}= & 1+\left(\sum_{x^{1}: 1 \leq w \leq N^{1 / 3}} \prod_{t=1}^{T}\left(1-L_{t} \sum_{k: x_{k}^{0} \neq x_{k}^{1}} p_{t, k}\right)\right)\left(1+O\left(\frac{w}{N}\right)^{2}\right)^{T}= \\
= & +\left(\sum_{w=1}^{\left[N^{1 / 3}\right]} \sum_{1 \leq k_{1}<\ldots<k_{w} \leq N} \prod_{t=1}^{T}\left(1-L_{t} \sum_{j=1}^{w} p_{t, k_{j}}\right)\right)(1+o(1))= \\
=1 & \left(\sum_{w=1}^{\left[N^{1 / 3}\right]} \sum_{1 \leq k_{1}<\ldots<k_{w} \leq N} \prod_{t=1}^{T} \prod_{j=1}^{w}\left(1-L_{t} p_{t, k_{j}}\right)\right)(1+o(1))= \\
= & +\left(\sum_{w=1}^{\left[N^{1 / 3}\right]} \frac{1}{w !} \sum_{k_{1}, \ldots, k_{w}=1}^{N} \prod_{j=1}^{w} \prod_{t=1}^{T}\left(1-L_{t} p_{t, k_{j}}\right)\right)(1+o(1))= \\
= & 1+\left(\sum_{w=1}^{\left[N^{1 / 3}\right]} \frac{1}{w !}\left(\sum_{k=1}^{N} \prod_{t=1}^{T}\left(1-L_{t} p_{t, k}\right)\right)\right)(1+o(1)) .
\end{aligned}
$$

ЗАМЕЧАНИЕ 4. Из условия $\mathbf{E} \xi=O(1)$, (35) и (36) следует, что

$$
\sum_{k=1}^{N} \prod_{t=1}^{T}\left(1-L_{t} p_{t, k}\right)=O(1) .
$$

Из этого равенства и (36) получаем:

$$
E_{1}=\exp \left\{\sum_{k=1}^{N} \prod_{t=1}^{T}\left(1-L_{t} p_{t, k}\right)\right\}+o(1) .
$$


ЗАМЕЧАниЕ 5 . Так как величины $L_{t}$ и $N p_{t, k}$ отделены от нуля (замечание 3 и условие (4)), то из равенства замечания 4 следует, что $N e^{-\gamma T N^{-1}}=$ $=O(1)$ при некоторой $\gamma>0$. Следовательно, $N \ln N=O(T)$, а с учетом условия $T=O(N \ln N)$ и $N \ln T=O(T)$.

Оценим сумму $E_{2}$. Используя (22), получаем:

$$
\begin{aligned}
E_{2} & \leq \sum_{x^{1}: N^{1 / 3}<w \leq \varepsilon N} \prod_{t=1}^{T}\left(1-L_{t}\left(1-\tilde{C} \frac{w}{N}\right) \sum_{k: x_{k}^{0} \neq x_{k}^{1}} p_{t, k}\right)< \\
< & \sum_{w=\left[N^{1 / 3}\right]}^{[\varepsilon N]} \sum_{1 \leq k_{1}<\ldots<k_{w} \leq N} \prod_{t=1}^{T}\left(1-L_{t}\left(1-\tilde{C} \frac{w}{N}\right) \sum_{j=1}^{w} p_{t, k_{j}}\right) \leq \\
& \leq \sum_{w=\left[N^{1 / 3}\right]}^{[\varepsilon N]} \frac{1}{w !} \prod_{j=1}^{w} \sum_{k_{j}=1}^{N} \prod_{t=1}^{T}\left(1-L_{t}\left(1-\tilde{C} \frac{w}{N}\right) p_{t, k_{j}}\right)= \\
& =\sum_{w=\left[N^{1 / 3}\right]}^{[\varepsilon N]} \frac{1}{w !}\left(\sum_{k=1}^{N} \prod_{t=1}^{T}\left(1-L_{t}\left(1-\tilde{C} \frac{w}{N}\right) p_{t, k}\right)\right)^{w} .
\end{aligned}
$$

Изучим, как зависят от номера ж слагаемые $\sigma_{w}$ внешней суммы в последнем выражении в (38). Для этого оценим отношение соседних слагаемых. Получаем (здесь $C_{1}, \ldots, C_{6}$ - некоторые конечные константы):

$$
\begin{aligned}
\frac{\sigma_{w+1}}{\sigma_{w}}= & \frac{\frac{1}{(w+1) !}\left(\sum_{k=1}^{N} \prod_{t=1}^{T}\left(1-L_{t}\left(1-\tilde{C} \frac{w+1}{N}\right) p_{t, k}\right)\right)^{w+1}}{\frac{1}{w !}\left(\sum_{k=1}^{N} \prod_{t=1}^{T}\left(1-L_{t}\left(1-\tilde{C} \frac{w}{N}\right) p_{t, k}\right)\right)^{w}}= \\
= & \frac{\left(\sum_{k=1}^{N} \prod_{t=1}^{T}\left(1-L_{t}\left(1-\tilde{C} \frac{w+1}{N}\right) p_{t, k}\right)\right)^{w}}{\left(\sum_{k=1}^{N} \prod_{t=1}^{T}\left(1-L_{t}\left(1-\tilde{C} \frac{w}{N}\right) p_{t, k}\right)\right)^{w}} \times \\
& \times \frac{1}{w+1} \sum_{k=1}^{N} \prod_{t=1}^{T}\left(1-L_{t}\left(1-\tilde{C} \frac{w+1}{N}\right) p_{t, k}\right) \leq \\
\leq & \frac{C_{1}}{w+1} \sum_{k=1}^{N} \prod_{t=1}^{T}\left(1-L_{t} p_{t, k}\right)\left(1+C_{2} \frac{w}{N^{2}}\right)^{T} \leq \\
\frac{C_{3}}{w+1} & \left(1+C_{2} \frac{w}{N^{2}}\right)^{T} \leq C_{4} \exp \left\{\ln N\left(C_{5} \frac{w}{N}-\frac{\ln w}{\ln N}\right)\right\} \leq \\
& \leq C_{6} \exp \left\{\ln N\left(C_{5} \frac{w}{N}-\frac{1}{3}\right)\right\} .
\end{aligned}
$$


При $\varepsilon<1 /\left(3 C_{5}\right)$ выражение в правой части этой цепочки неравенств стремится к нулю. Значит, при таком $\varepsilon$ каждый член суммы в правой части (38) бесконечно мал по сравнению с предыдущим. Поэтому основной вклад в эту сумму вносит слагаемое с $w=\left[N^{1 / 3}\right]$. Убедимся, что оно также стремится к нулю.

Заметим, что в силу (37) и (4)

$$
\begin{gathered}
\sum_{k=1}^{N} \prod_{t=1}^{T}\left(1-L_{t}\left(1-\tilde{C} \frac{w}{N}\right) p_{t, k}\right)= \\
=\sum_{k=1}^{N} \prod_{t=1}^{T}\left(1-L_{t} p_{t, k}\right)\left(1+\frac{\tilde{C} L_{t} w p_{t, k}}{N\left(1-L_{t} p_{t, k}\right)}\right) \leq C_{1}\left(1+\frac{C_{2} w}{N^{2}}\right)^{T}
\end{gathered}
$$

при некоторых $C_{1}, C_{2}<\infty$. Кроме этого, воспользуемся известным неравенством $n !>(n / e)^{n}$, из которого следует, что

$$
\ln \left[N^{1 / 3}\right] !>N^{1 / 3}\left(\ln N^{1 / 3}-2\right)
$$

при достаточно больших $N$. Получаем:

$$
\begin{gathered}
\sigma_{\left[N^{1 / 3}\right]} \leq \frac{1}{\left[N^{1 / 3}\right] !}\left(C_{1}\left(1+\frac{C_{2} w}{N^{2}}\right)^{T}\right)^{N^{1 / 3}}< \\
<\exp \left\{N^{1 / 3}\left(T \ln \left(1+\frac{C_{2} \varepsilon}{N}\right)-\frac{1}{3} \ln N+\ln C_{1}+2\right)\right\} .
\end{gathered}
$$

При достаточно малом $\varepsilon$ и при $T=O(N \ln N)$ показатель этой экспоненты стремится к $-\infty$. Тогда первое слагаемое суммы (38) и сама величина $E_{2}$ стремятся к нулю.

Оценим сумму $E_{3}$. Согласно лемме 2 при $x^{1} \in \Sigma_{3}$ величина $P_{t}\left(x^{1}\right)$ отделена от единицы равномерно по $t$ и $x^{1}: P_{t}\left(x^{1}\right)<1-c(\varepsilon)$. Поэтому при достаточно больших $N$

$$
E_{3}<\sum_{x^{1}}(1-c(\varepsilon))^{T} \leq 2^{N}(1-c(\varepsilon))^{T} .
$$

Теперь из соотношения $N \ln N=O(T)$ (см. замечания 1 и 5) следует, что $E_{3} \rightarrow 0$.

Оценим сумму $E_{4}$. Сначала рассмотрим случай, когда выполнено условие $(A)$. Тогда согласно лемме 3 при $x^{1} \in \Sigma_{4}$, по крайней мере, при $M(N)$ 
значениях $t$ из диапазона $1 \leq t \leq T$ величина $P_{t}\left(x^{1}\right)$ отделена от единицы равномерно по $t$ и $x^{1}: P_{t}\left(x^{1}\right)<1-c^{\prime}(\varepsilon)$. Поэтому при достаточно больших $N$

$$
E_{4}<\sum_{x^{1} \in \Sigma_{4}}\left(1-c^{\prime}(\varepsilon)\right)^{M(T)}<2^{N}\left(1-c^{\prime}(\varepsilon)\right)^{M(T)} .
$$

Теперь из соотношений $M(T) \ln T / T \rightarrow \infty$ (условие $A$ ) и $N \ln T=O(T)$ (замечание 5) следует, что $M(T) N^{-1} \rightarrow \infty$. Значит, при условии $(A)$ имеет место соотношение $E_{4} \rightarrow 0$.

Рассмотрим случай, когда выполнено условие $(B)$. Тогда наряду с заданным решением $x^{0}$ система (6) имеет решение $\bar{x}^{0}=\left(1-x_{1}^{0}, \ldots, 1-x_{N}^{0}\right)$. Положим

$$
\begin{gathered}
\bar{\varpi}=\bar{\varpi}\left(x^{1}\right)=\left|\left\{k: x_{k}^{1} \neq \bar{x}_{k}^{0}\right\}\right|, \\
\bar{\Sigma}_{1}=\left\{x^{1}: \bar{\varpi} \leq N^{1 / 3}\right\}, \quad \bar{\Sigma}_{2}=\left\{x^{1}: N^{1 / 3}<\bar{\varpi} \leq \varepsilon N\right\} .
\end{gathered}
$$

Аналогичным образом определим суммы $\bar{E}_{1}$ и $\bar{E}_{2}$.

Очевидно, что $\Sigma_{4}=\bar{\Sigma}_{1} \cup \bar{\Sigma}_{2}, \bar{E}_{1}=E_{1}, \bar{E}_{2}=E_{2}$. Поэтому $E_{4}=E_{1}+E_{2}$. Значит, в этом случае из доказанного выше соотношения $E_{2} \rightarrow 0$ следует, что $E_{4}=E_{1}+o(1)$.

Таким образом, согласно (35) и (37) доказано, что при условии $(A)$ справедливо соотношение

$$
\mathbf{E} \xi=\exp \left\{\sum_{k=1}^{N} \prod_{t=1}^{T}\left(1-L_{t} p_{t, k}\right)\right\}+o(1),
$$

а при условии $(B)$ справедливо соотношение

$$
\mathbf{E} \xi=2 \exp \left\{\sum_{k=1}^{N} \prod_{t=1}^{T}\left(1-L_{t} p_{t, k}\right)\right\}+o(1) .
$$

Из (2)-(5) и замечания 3 следует, что $L_{t} p_{t, k}=O\left(N^{-1}\right)$ равномерно по $t$ и $k$. Поэтому

$$
\begin{gathered}
-\ln \prod_{t=1}^{T}\left(1-L_{t} p_{t, k}\right)=\sum_{t=1}^{T} L_{t} p_{t, k}+O\left(\sum_{t=1}^{T} L_{t}^{2} p_{t, k}^{2}\right)= \\
=\sum_{t=1}^{T} L_{t} p_{t, k}\left(1+O\left(\frac{1}{N}\right)\right)
\end{gathered}
$$


равномерно по $k$. Согласно формуле (14) выражение $L_{t} p_{t, k}$ с точностью до множителя $1+O\left(N^{-1}\right)$ (равномерно по $k$ и $t$ ) равно вероятности $a_{t, k}$ того, что переменная $x_{k}$ присутствует и не допускает варьирования в $t$-м уравнении. Поэтому из (8) и (42) следует равенство

$$
\exp \left\{\sum_{k=1}^{N} \prod_{t=1}^{T}\left(1-L_{t} p_{t, k}\right)\right\}=e^{\lambda_{T, N}\left(1+O\left(N^{-1}\right)\right)} .
$$

Из (40), (41) и (43) следуют соотношения (33) и (34). Лемма 4 доказана.

\section{5. Доказательства теорем}

Доказательство теоремы 1. Из соотношения (7) следует (см., например, [4]), что

$$
\underline{\lim }\left(\mathbf{E} \xi^{\prime}-e^{\lambda_{T, N}}\right) \geq 0 .
$$

При условии $(A)$ воспользуемся соотношениями $\mathbf{E} \xi^{\prime} \leq \mathbf{E} \xi$ и $\mathbf{P}\left\{\xi^{\prime} \leq \xi\right\}=1$. Благодаря им из (33) и (44) получаем, что

$$
\lim \mathbf{E} \xi=\lim \mathbf{E} \xi^{\prime} \quad \text { и } \quad \mathbf{P}\left\{\xi \neq \xi^{\prime}\right\} \rightarrow 0 .
$$

При условии $(B)$ помимо решений, близких к вектору $x^{0}$, система (6) имеет еще столько же решений, близких к вектору $\bar{x}^{0}$. Поэтому выполняются соотношения $2 \mathbf{E} \xi^{\prime} \leq \mathbf{E} \xi$ и $\mathbf{P}\left\{2 \xi^{\prime} \leq \xi\right\}=1$. Теперь из (34) и (44) получаем, что

$$
\lim \mathbf{E} \xi=\lim 2 \mathbf{E} \xi^{\prime} \quad \text { и } \quad \mathbf{P}\left\{\xi \neq 2 \xi^{\prime}\right\} \rightarrow 0 .
$$

Осталось воспользоваться соотношением (7). Теорема 1 доказана.

Теорема 2 вытекает непосредственно из теоремы 1, соотношения (7) и условия $\mu=\lim \lambda_{T, N}$.

Автор признателен А. М. Зубкову за поддержку и полезные замечания.

\section{Список литературы}

1. Балакин Г.В. Введение в теорию случайных систем уравнений. - В сб.: Труды по дискретной математике. Т. 1. - М.: ТВП, 1997, с. 1-18.

2. Копытиев B.A. О распределении числа решений случайных заведомо совместных систем уравнений. - Теория вероятн. применен., 1995, т. 40, в. 2 , c. $430-437$.

3. Копытиев В.А. Предельные теоремы для числа решений системы случайных уравнений. - Теория вероятн. применен., 2000, т. 45, в. 1, с. 51-72. 
4. Михайлов В.Г. Предельные теоремы для случайного покрытия конечного множества и для числа решений системы случайных уравнений. - Теория вероятн. применен., 1996, т. 41, в. 2, с. 272-283.

5. Михайлов В.Г. Сведение задачи о предельном поведении числа решений системы уравнений со случайным вхождением неизвестных к одной задаче о размещении частиц. - В сб.: Труды по дискретной математике. Т. 11, в. 2. - М.: ФИЗМАТЛИТ, 2008, с. 112-124. 